\title{
Ultrasonic Nondestructive Evaluation and Piezoelectric Materials
}

Of the different nondestructive evaluation techniques, ultrasonic probes have a distinct advantage-ultrasonic waves have no residual physical effect on the material being analyzed. Ultrasonic echoes and shadows transmitted through materials have been used since the end of World War II to detect cracks, nonbonds, inclusions, and interfaces.

Though at first the technique seemed to have questionable practicality, other ultrasonic applications developed in tandem with the emergence of new piezoelectric materials that were crucial for creating ultrasonic waves. This Historical Note will trace the use of ultrasonic testing methods and the development of piezoelectric materials.

The term "ultrasonic" is used for mechanical vibrational waves at frequencies beyond the range of human hearing (above $20 \mathrm{kHz}$ ). Until about 1935, the field was known as "supersonics," but confusion arose with the study of fluid-flow velocities exceeding the speed of sound in a material, which was also known as supersonics. Since then, the term "ultrasonics" has been adopted for high-frequency sound waves.

Ultrasonic waves themselves were known by 1910 but viewed as little more than a laboratory curiosity. In World War I, though, highly directional ultrasonic beams (made possible by the development of the first piezoelectric transducer, as described below) were used as a crude form of sonar to detect enemy submarines. Sonar is an acronym for Sound Navigation and Ranging.

In World War II, sonar and ultrasonic detection systems worked in tandem with the newly developed radar, which could detect aircraft and surface ships, while sonar detected submarines. Ultrasonic waves were also used in different applications, such as attempts at communication transmission and as light modulators in the first experiments with television.

In April 1942 F.A. Firestone patented the "ultrasonic reflectoscope," which used ultrasonic waves to probe the material structure of metals and other materials. Firestone's reflectoscope sent a penetrating ultrasonic pulse into a material, which produced an echo when it struck a flaw bearing different acoustic properties than the surrounding substance. These echoes could then be detected by the same transducer that emitted the signal. Similarly, an ultrasonic beam striking an anomaly would create a shadow detectable by a transducer on the opposite side. Ultrasonic inspection of metals was particularly important because electromagnetic beams used in a similar fashion for inspection would have been readily absorbed by any conductor.

Because of their very short wavelengths, ultrasonic beams can produce much sharper shadows and echoes than lower frequency acoustic waves. The ultrasonic frequency selected for nondestructive evaluation usually falls between 1 and $15 \mathrm{MHz}$, depending on the material, its elastic modulus, its grain size, and the necessary sensitivity of the measurement.

It was found that selecting the proper frequency and type of wave allowed investigation of any material that transmits vibrational energy. Based on its shear or elastic modulus and its density, each such material displays a characteristic sound velocity.

Because of the low attenuation of ultrasonic waves in liquids and solids, materials up to 30 feet thick can be tested by either pulsed or continuous waves. Metals, weldings, castings, and forgings can be investigated for internal flaws, porosity, or inclusions, as well as fatigue cracks in machinery. Noncellular plastics, ceramics, glass, new concrete, organic materials, and rubber can be similarly tested. Nuclear fuel elements can be investigated for unbonding.

In the 1960s, many applications for ultrasonics came into practice; some were unsuccessful while others are still used today. Ultrasonics were used to clean delicate machinery, and to agitate abrasive pastes to make industrial drills to cut holes of any shape in brittle substances. The same principle was even investigated for dental drills. Ultrasonics were also used for making fine measurements of material layers; this application was adopted for medical uses, such as measuring the thickness of a heart wall in a living patient.

In Sweden, one ultrasonic unit was tested for defoaming, drying, and processing aerosol solutions. Some airports conducted research in using ultrasonics for fume precipitation (i.e., fog dissipation), but the large energy requirements to run such a system proved impractical. One London dairy even experimented with ultrasonics to improve the quality of pasteurized milk.

Railroads still use ultrasonic testing methods to check their rail systems for flaws and deformations that might cause accidents. In 1962 the city of Detroit, Michigan, installed an ultrasonic sensor system for traffic control and surveillance.

\section{Piezoelectric Materials}

While the concept of ultrasonic testing may seem simple, the difficulties of actually generating ultrasonic waves delayed its adoption for some time. Eventually, the development of a new class of materials, piezoelectric materials, was required before the method could be used.

The first method found for generating ultrasonic vibrations used magnetostrictive effects. As far back as 1846, James Prescott Joule had discovered that a ferromagnetic bar expands when it is in a weak magnetic field, but contracts again once it has reached magnetic saturation. Known as the Joule effect, this was investigated more extensively by George Washington Pierce. Applying a high-frequency voltage to a solenoid coil around a magnetized metal rod can produce mechanical vibrations from 20 to $175 \mathrm{kHz}$, which can be used for ultrasonic testing.

A more popular method for generating ultrasonic waves uses piezoelectric materials. Piezo comes from the Greek, meaning "to press"; in response to mechanical pressure, piezoelectric materials generate an electric pulse. Conversely, such materials oscillate mechanically when subjected to an oscillating voltage. A transducer made from a piezoelectric material is used as an ultrasonic generator for either longitudinal or shear waves, depending on how the transducer is made.

Pierre and Paul-Jacques Curie discovered the piezoelectric effect in 1880 while they were both young researchers at the University of Paris (Pierre was 21, Paul-Jacques was 24). After extensive theoretical and experimental study of the symmetry of crystals, the Curies found that by placing a weight on a crystal they could observe a voltage on the surface of the crystal. 
A year later, they verified the converse effect, that applying a voltage to the crystal created a mechanical elongation. Further testing showed the same effect on many different asymmetrical crystals, including quartz, tourmaline, and Rochelle salt (sodium potassium tartrate).

An oscillating voltage applied to these crystals created ultrasonic waves. In France in 1916, Paul Langevin sandwiched a piezoelectric quartz element between two steel plates; as an ultrasonic source, this device was submerged under water and the echoes used to detect hidden submarines in World War I. This crude sonar system was refined and eventually became practical.

Further experimentation during the war caused Walter G. Cady of Wesleyan University to recognize how a vibrating crystal's mechanical resonance affects its electrical behavior, which led to frequency control for oscillators. This breakthrough led to the wide application of piezoelectric crystals for controlling frequencies in radio-communication systems. During World War II alone, the United States used 50 million quartz-crystal elements. Closely related to this was the use of piezoelectric crystals as wave filters for multichannel telephone systems, developed at Bell Telephone Laboratories around 1925.

Because it can be easily cut to preferred orientations, quartz is the most popular piezoelectric crystal. The oscillations produced by quartz crystals are stable and virtually temperature independent. Brazil maintained a near monopoly on the highquality quartz crystals needed for ultrasonic applications. It was not until 1958 that an industrial process for synthetically growing acceptable quartz crystals alleviated this dependence.

Rochelle salt, one of the first identified piezoelectric crystals, was originally called Seignette salt, after its French discoverer P. Seignette, an apothecary who discovered medical uses of the salt in 1672 . When Russian researchers studied the crystal extensively during the $1930 \mathrm{~s}$, they called the piezoelectric effects "Seignette electricity." In 1945, however, it was recognized that Rochelle salt and some other piezoelectric materials actually exhibit a similar phe- nomenon, termed "ferroelectricity" because their dielectric properties are very analogous to the ferromagnetic properties of iron. Ferroelectric crystals have a permanent spontaneous electric polarization that can be reversed by an electric field. All ferroelectric crystals are also piezoelectric materials, though the converse is not necessarily true. The ferroelectric properties of Rochelle salt are very temperature sensitive, however, operating only from $-18^{\circ} \mathrm{C}$ to $+24^{\circ} \mathrm{C}$

In the early 1940 s the search began for other crystals that exhibited the same piezoelectric properties as Rochelle salt, but did not suffer from its temperature limitations or its dielectric anomalies. Among the primary investigators was Bell Laboratories, who by 1950 had tested about 500 different crystals, many of which were found to have piezoelectric effects. The most successful direct results of this search were ammonium dihydrogen phosphate (ADP), ethylene diamine tartrate (EDT), and dipotassium tartrate (DKT), which replaced quartz crystals in telephone filters, the largest application of piezoelectric crystals in the telephone system. Especially popular was ADP, which is even more piezoelectrically sensitive than quartz and more rugged than Rochelle salt, since it has no water of crystallization.

Newly developed crystals such as lithium niobate and lithium tantalate are also being used to replace quartz in the frequency control of radio transmitters and as wave filters for telephone communications.

In 1940 Arthur von Hippel and his coworkers at the Massachusetts Institute of Technology discovered that certain ceramics have ferroelectric properties. Polycrystalline ceramic barium titanate, the first such material to be identified, was found to have a dielectric constant higher than 1,000 . Treatment in a high electric field at a temperature near the "Curie point" (the transition point below which a material becomes ferroelectric) could permanently reverse the direction of polarity in the crystallites of a ceramic, polycrystalline body of barium titanate, which caused it to become strongly piezoelectric when it cooled.
Barium titanate could compare with Rochelle salt in piezoelectric sensitivity, and also with quartz in its chemical stability. Because of ceramic preparation methods, the material could be molded into desired shapes and sizes unattainable with single crystals. The cylindrical shape became the most popular detector for nondirectional reception. In other uses, a spherically shaped cup of barium titanate could be used to concentrate ultrasonic waves as a transducer.

Above $120^{\circ} \mathrm{C}$, though, barium titanate loses its ferroelectric and piezoelectric properties (by comparison, quartz crystals remain stable to $575^{\circ} \mathrm{C}$ ). In the 1950 s it was found that adding small amounts of lead and calcium titanate to the ceramic would raise its Curie temperature. Also about the same time, piezoelectric effects were found in ceramic lead metaniobate and lead titanate zirconate, which retain their piezoelectric properties to $250^{\circ} \mathrm{C}$. By the late 1950s these ceramic piezoelectric materials had nearly replaced Rochelle salt and other crystalline materials, except for quartz.

The development of the laser in the 1960 s created a new demand for crystalline piezoelectric materials, such as ammonium dihydrogen phosphate and potassium dihydrogen phosphate. All piezoelectric crystals exhibit the electrooptical effect-a change in optical properties with the application of a voltage. Using piezoelectric crystals as light modulators, laser communication systems can transmit a large number of communications channels over a single beam. The laser also allowed attaining the highest frequency ultrasonic beam: Use of a ruby laser on a sapphire crystal increased the previous maximum of $10,000 \mathrm{MHz}$ to $60,000 \mathrm{MHz}$.

The field of ultrasonics has branched out widely from nondestructive evaluation and communications applications to extensive use in medical techniques and delicate biological research. The development of new piezoelectric materials and the discovery of new applications of ultrasonics has not slowed, suggesting that many new possibilities remain to be uncovered.

KEVIN J. ANDERSON

\title{
Equipment Exhibit at the 1990 MRS Spring Meeting...
}

\author{
See p. 51
}

